\title{
Data fusion for reconstruction algorithms via different sensors in geophysical sensing
}

\author{
Sven Nordebo (1), Mats Gustafsson (2), and Francesco Soldovieri (3) \\ (1) School of Computer Science, Physics and Mathematics, Linnaeus University, Växjö, Sweden (sven.nordebo@lnu.se), (2) \\ Department of Electrical and Information Technology, Lund University, Lund, Sweden (mats.gustafsson@eit.lth.se), (3) \\ Institute for Electromagnetic Sensing of the Environment, National Research Council, Napoli, Italy (soldovieri.f@irea.cnr.it)
}

\section{Abstract}

A unified approach to sensitivity analysis and data fusion for multi-physics inverse scattering problems will be presented which is based on Fisher information analysis for distributed parameter systems, cf., [4]. This approach is particularly useful when there is a diversity of measurement sensors having varying noise spectral content and signal to noise ratios, such as with information fusion with multi-physics data. Here, the Fisher information analysis yields a systematic procedure to incorporate a statistically based weighting of the measurements.

The forward operator is the mapping that takes the unknown material parameters of interest to the measurement data. It will be shown that the Fisher information analysis is equivalent to performing a Singular Value Decomposition (SVD) analysis of the linearized forward operator [1,2,5], provided that the measurement noise is Gaussian distributed and that the appropriate scalar product is chosen for the space of measurement data. Hence, the Fisher information concept provides a systematic means of choosing the scalar product for the related Hilbert space in a way that is statistically optimum.

In particular, if $\mathcal{J}$ denotes the Jacobian, or the Fréchet derivative of the forward operator, then the Fisher information integral operator is given by $\mathcal{I}=2 \mathcal{J}^{*} \mathcal{J}$ where $\mathcal{J}^{*}$ is the Hilbert adjoint operator, see e.g., [3] for the finite-dimensional case. Hence, the truncated SVD algorithm [1,2,5] corresponds here to a regularized pseudoinverse based on the Fisher information operator.

Application examples using the truncated SVD algorithm [5] for multi-physics inverse problems in geophysical sensing will be considered.

\section{References}

[1] M. Bertero. Linear inverse and ill-posed problems. Advances in electronics and electron physics, 75, 1-120, 1989.

[2] Q. Fang, P. M. Meaney, and K. D. Paulsen. Singular value analysis of the Jacobian matrix in microwave image reconstruction. IEEE Trans. Antennas Propagat., 54(8), 2371-2380, aug 2006.

[3] S. M. Kay. Fundamentals of Statistical Signal Processing, Estimation Theory. Prentice-Hall, Inc., NJ, 1993.

[4] S. Nordebo, A. Fhager, M. Gustafsson, and B. Nilsson. Fisher information integral operator and spectral decomposition for inverse scattering problems. Technical Report LUTEDX/(TEAT-7180)/1-21/(2009), Lund Institute of Technology, Department of Electrical and Information Technology, P.O. Box 118, S-211 00 Lund, Sweden, 2009. Submitted to Inverse Problems in Science and Engineering.

[5] R. Pierri, A. Liseno, and F. Soldovieri. Shape reconstruction from PO multifrequency scattered fields via the singular value decomposition approach. IEEE Trans. Antennas Propagat., 49(9), 1333-1343, September 2001. 